\title{
Autism Spectrum Disorder in an Unselected Cohort of Children with Neurofibromatosis Type 1 (NF1)
}

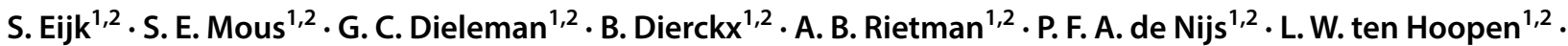 \\ R. van Minkelen ${ }^{2,3} \cdot$ Y. Elgersma ${ }^{2,4} \cdot$ C. E. Catsman-Berrevoets ${ }^{2,5} \cdot$ R. Oostenbrink ${ }^{2,6} \cdot$ J. S. Legerstee ${ }^{1,2,7}$
}

Published online: 8 February 2018

(c) The Author(s) 2018. This article is an open access publication

\begin{abstract}
In a non-selected sample of children with Neurofibromatosis type 1 (NF1) the prevalence rate of autism spectrum disorder (ASD) and predictive value of an observational (ADOS) - and questionnaire-based screening instrument were assessed. Complete data was available for 128 children. The prevalence rate for clinical ASD was $10.9 \%$, which is clearly higher than in the general population. This prevalence rate is presumably more accurate than in previous studies that examined children with NF1 with an ASD presumption or solely based on screening instruments. The combined observational- and screening based classifications demonstrated the highest positive predictive value for DSM-IV diagnosis, highlighting the importance of using both instruments in children with NF1.
\end{abstract}

Keywords Neurofibromatosis type $1 \cdot$ Autistic traits $\cdot$ Autism spectrum disorder $\cdot$ Prevalence $\cdot$ Autism diagnostic observation schedule $\cdot$ Social responsiveness scale

\section{Introduction}

$\bowtie$ J. S. Legerstee

j.s.legerstee@erasmusmc.nl

1 Department of Child and Adolescent Psychiatry/Psychology, Erasmus Medical Center-Sophia Children's Hospital, P.O. Box 2060, 3000 CB Rotterdam, The Netherlands

2 ENCORE Expertise Center for Neurodevelopmental Disorders, Erasmus Medical Center Sophia Children's Hospital, P.O. Box 2060, 3000 CB Rotterdam, The Netherlands

3 Department of Clinical Genetics, Erasmus Medical Center, P.O. Box 2040, 3000 CA Rotterdam, The Netherlands

4 Department of Neuroscience, Erasmus Medical Centre Rotterdam, 3015 CN Rotterdam, The Netherlands

5 Department of Pediatric Neurology, Erasmus Medical Center-Sophia Children's Hospital, P.O. Box 2060, 3000 CB Rotterdam, The Netherlands

6 Department of General Paediatrics, Erasmus Medical Center-Sophia Children's Hospital, P.O. Box 2060, 3000 CB Rotterdam, The Netherlands

7 Department of Child and Adolescent Psychiatry/Psychology, Erasmus Medical Center-Sophia Children's Hospital, Room Sp-2509, P.O. Box 2060, 3000 CB Rotterdam, The Netherlands
Neurofibromatosis type 1 (NF1) is an autosomal dominant disorder affecting 1 in 2500-3000 individuals (Williams et al. 2009). The disorder is inherited in half of the cases, and in the other half the mutation is de novo (Messiaen et al. 2000). The NF1 gene encodes for the protein neurofibromin, which activates the protein RasGTPase (Rauen 2013). RasGTPase functions as a negative regulator of Ras, a protein involved in the regulation of the cell cycle, growth and differentiation. As a result of mutations in NF1, a decrease in neurofibromin activity causes increased cell growth. Affected individuals are recognized by the representation of at least two distinctive physical features, including caféau-lait spots, intertriginous freckling, Lisch nodules, neurofibromas, optic pathway gliomas or distinctive bone-forming lesions (Williams et al. 2009).

Children with NF1 often experience cognitive and behavioral problems (Hachon et al. 2011; Lehtonen et al. 2013). Generally, intelligence scores of affected children are significantly lower compared to the general population, and learning problems and attention-deficit-hyperactivity disorder (ADHD) are common. However, the problems are highly variable across the NF1 population (Lehtonen et al. 2013). Besides these common cognitive and behavioral 
characteristics, social difficulties have been reported in children with NF1. Children with NF1 often have poorer social skills, tend to be socially isolated and rejected by peers, and experience problems in social information processing (Barton and North 2004; Huijbregts et al. 2010; Noll et al. 2007). Similar to the general population, social problems in children with NF1 are more prevalent in boys (Garg et al. 2016) and in children with low intellectual functioning (Huijbregts and De Sonneville 2011).

Recently, studies have focused on the prevalence and profile of autism spectrum disorders (ASD) in children with NF1. Compared to global ASD prevalence estimates of $0.8 \%$ in the general population (Baxter et al. 2015) screening-based prevalence rates of clinical ASD symptoms of 13-29\% have been found in children with NF1. On top of this, an additional percentage of $27-31 \%$ of children were found to show subclinical symptoms, leading to total estimated screening-based prevalence rates of ASD symptoms ranging between 30-56\% (Adviento et al. 2014; Constantino et al. 2015; Garg et al. 2013; Van Eeghen et al. 2013; Walsh et al. 2013). In a recent, internationally compiled sample of children with NF1 $(N=531)$, a screening-based prevalence rate of clinical ASD of $13 \%$ and an additional prevalence rate of $26 \%$ of subclinical symptoms was reported (Morris et al. 2016), resulting in a total prevalence rate of ASD traits of $39 \%$. It should be noted, though, that these estimates are based on screening instruments. Because of their measurement purposes, these instruments are highly sensitive and thus may result in a biased (slightly overestimated) ASD prevalence rate.

The possible overestimation of the screening-based prevalence rates highlights the importance of studies assessing clinical ASD prevalence rates. Only a few studies have examined clinically assessed ASD prevalence rates in children with NF1. In the studies by Garg et al. (2013) and Plasschaert et al. (2015), children with a presumption of ASD (preselected based on elevated scores on a screening instrument) were assessed with clinical diagnostic instruments. In these subsamples of children with NF1, ASD prevalence estimates of 25\% (Garg et al. 2013) and 26\% (Plasschaert et al. 2015) were reported. Because these prevalence rates were based on samples of children with an initial suspicion of autism spectrum problems, these prevalence rate are probably not representative for the general pediatric NF1 population as a whole. To our knowledge, there are no reports available in the literature in which an unselected sample of children with NF1 has been clinically assessed for ASD.

The primary aim of this study was to examine the prevalence of clinically assessed ASD in children with NF1 visiting a specialized NF1 outpatient clinic without a presumption of ASD. The secondary aim was to investigate the predictive value of a screening instrument and an observational assessment in relation to clinical DSMIV ASD diagnosis in a pediatric NF1 population. Also, the association of gender, age and intellectual functioning with ASD diagnosis was examined.

\section{Method}

\section{Participants}

Eligible for participation were children (aged 2-10) with either genetically or clinically diagnosed NF1. All children were patients of ENCORE, a multidisciplinary expertise center for genetic neurocognitive disorders (including NF1) in Rotterdam. As part of the standard multidisciplinary care for and follow-up of children with NF1, these children were routinely referred to the Department of Child and Adolescent Psychiatry/Psychology, between August 2011 and August 2016. In the current study, a total of 128 children between 2 and 10 years of age with NF1 were enrolled (45.3\% female, mean age $=5.27, S D=1.81$ ).

\section{Procedure}

As standard procedure, all children underwent neuropsychological evaluation and clinical assessment of autistic symptomatology. Additionally, parents and teachers provided information concerning the child's development and the primary caregiver was asked to complete several questionnaires, including the SRS. The data in this prospective study was collected based on a fixed protocol in the context of the longitudinal follow-up for the assessment of clinical symptoms in children with NF1.

\section{Measures}

\section{ASD Symptom Screening}

ASD symptoms were screened with the social responsiveness scale (SRS) (Constantino et al. 2003). Completion of the 65 items by one of the parents provides information concerning functioning in the domains social awareness, social cognition, reciprocal social communication, social motivation, and autistic mannerisms. The total raw score, the sum of the 65 items, can be converted into a T-score $(M=50 ; S D=10)$ using a Dutch normative reference group (Constantino and Gruber 2015). T-scores of 60 or higher indicate mild to moderate problems, and T-scores of 76 or more indicate severe (clinical) problems. The SRS has been 
shown to be a valid and reliable instrument the scores are independent from IQ scores (Constantino et al. 2003).

\section{Observational Assessment of ASD}

Observational assessment of ASD was carried out with the autism diagnostic observation schedule-generic (ADOS-G) (Lord et al. 1999) and the autism diagnostic observation schedule-second edition (ADOS-2)(Lord et al. 2012). In most cases (i.e. 88.3\%), the ADOS-2 was used. With the ADOS, social interaction, play and imaginative skills are assessed. The ADOS was performed by trained and certified psychologists. Depending on the developmental age and level of expressive language of the child, one of the four available modules of the ADOS was chosen. The ADOS has been shown to be a reliable and valid measure for ASD symptoms (Gotham et al. 2007).

ADOS-G scores were converted to ADOS-2 scores according to the manual (Lord et al. 2012). ASD classifications were obtained and to enable comparison between ASD severity across the four different modules, continuous calibrated severity scores (CSS) were calculated (Gotham et al. 2009; Hus et al. 2014; Hus and Lord 2014). The CSS range from zero to ten with zero indicating no or very little symptoms and ten indicating severe ASD symptoms.

\section{Clinical (DSM-IV) Diagnosis}

A clinical DSM-IV diagnosis of ASD was established by a multidisciplinary team consisting of a child and adolescent psychiatrist and psychologists, combining information from all assessments, questionnaires, observation of the child and heteroanamnestic information provided by parents and teachers.

\section{Intellectual Functioning}

Depending on the child's age, the level of intellectual functioning was assessed with either the wechsler preschool and primary scale of intelligence (WPPSI-III)(Wechsler 2002) or the wechsler intelligence scale for children-iii (WISC-III) (Wechsler 1991). Reliability and validity of these intelligence tests have been demonstrated. Standardized verbal-, performance-, and full scale IQ scores were calculated $(M=100, S D=15)$. For one child, a nonverbal intelligence test (i.e. the Wechsler Non Verbal scale of Ability; WNV) (Wechsler and Naglieri 2006) was used, for which the total IQ score was calculated as well. In two children, assessment with the Wechsler scales was not possible due to a developmental delay. In these children, assessment of intellectual functioning was done using the Bayley Scales of Infant and
Toddler Development third edition (Bayley 2006), and a developmental quotient was calculated (developmental age/ chronological age $\times 100$, with $M=100, S D=15$ ).

\section{Statistical Analyses}

To study the prevalence of ASD, frequencies of the SRS classifications, ADOS-2 classifications, and clinically derived DSM-IV diagnosis were calculated. Sensitivity, specificity, positive predictive (PPV) and negative predictive values (NPV) were calculated to assess the screening accuracy of the instruments' classifications. The association of intelligence and age with clinically derived DSM-IV ASD diagnosis was examined with independent $t$ tests. Missing full-scale IQ scores were imputed using mean imputation. The association of gender with clinically derived DSM-IV ASD diagnosis was examined with a Chi square test. Data were analyzed using IBM SPSS Statistics version 22. Results were considered statistically significant if the (two-tailed) alpha level was below .05. Sensitivity, specificity, PPV and NPV values were interpreted according to the guidelines presented by Cicchetti (2001).

\section{Results}

\section{Sample Characteristics}

Patient characteristics are summarized in Table 1. Of the total sample of 128 children, 58 were female $(45.3 \%)$. The mean age at assessment of the sample was 5.27 years $(S D=1.81)$. Intelligence scores were significantly lower than the general population mean of $100(S D=15$; total IQ $t(122)=-9.40, p<.001$, verbal IQ $t(123)=-6.77$, $p<.001$, performance IQ $t(124)=-9.24, p<.001)$. Mutations in NF1 were detected as described before (Van Minkelen et al. 2014). The mutations were familiarly inherited in $17.2 \%(N=22)$ of the children and de novo in $41.4 \%(N=53)$ of the children. In $41.4 \%$ of the cases it was unknown whether the mutation was familial or de novo, caused by the fact that parents were not genetically tested, one of the parents was not genetically tested, or the child was not genetically tested yet. The SRS questionnaire was completed by the primary caregiver in 103 children. The mean SRS total T-score was $54.7(S D=12.60)$, which is significantly higher compared to the general population mean of $50(S D=10)$, $t(102)=3.79, p<.001$. There were no significant differences between the group of children with and without available SRS scores regarding gender, intelligence scores, age, ADOS CSS or DSM-IV diagnosis. 
Table 1 Patient characteristics

\begin{tabular}{|c|c|c|c|c|}
\hline & $N(\%)$ & Mean $(S D)$ & Min & $\operatorname{Max}$ \\
\hline Age at assessment (years) & 128 & $5.27(1.81)$ & 2 & 10 \\
\hline Female gender & $58(45.3 \%)$ & & & \\
\hline \multicolumn{5}{|l|}{ Mutation } \\
\hline Familial & $22(17.2 \%)$ & & & \\
\hline De novo & $53(41.4 \%)$ & & & \\
\hline Unknown & $53(41.4 \%)$ & & & \\
\hline \multicolumn{5}{|l|}{ ADOS modules } \\
\hline Module 1 & $12(9.4 \%)$ & & & \\
\hline Module 2 & $45(35.2 \%)$ & & & \\
\hline Module 3 & $71(55.5 \%)$ & & & \\
\hline \multicolumn{5}{|l|}{$\begin{array}{l}\text { ADOS calibrated severity } \\
\text { score }\end{array}$} \\
\hline Total & 128 & $2.34(1.83)$ & 1 & 8 \\
\hline Social affect & 128 & $2.70(2.12)$ & 1 & 9 \\
\hline $\begin{array}{l}\text { Restricted/repetitive } \\
\text { behaviors }\end{array}$ & 128 & $3.34(2.50)$ & 1 & 10 \\
\hline \multicolumn{5}{|l|}{ Type of intelligence test } \\
\hline WPPSI & $98(76.5 \%)$ & & & \\
\hline WISC-III & $27(21.1 \%)$ & & & \\
\hline WNV-III & $1(.8 \%)$ & & & \\
\hline BSID-III & $2(1.6 \%)$ & & & \\
\hline \multicolumn{5}{|l|}{ IQ scores } \\
\hline Full-scale IQ & 123 & $88.20(13.93)$ & 55 & 119 \\
\hline Verbal IQ & 124 & $91.64(13.75)$ & 57 & 124 \\
\hline Performance IQ & 125 & $88.25(14.21)$ & 58 & 130 \\
\hline \multicolumn{5}{|l|}{ SRS } \\
\hline Total T-score & 103 & $54.70(12.59)$ & 35 & 103 \\
\hline
\end{tabular}

\section{Autism Prevalence}

Of the 128 children with NF1 included in the analyses, 14 received a clinical DSM-IV ASD diagnosis, resulting in a prevalence rate of $10.9 \%$. Based on the SRS, $8.7 \%$ of the patients achieved scores in the 'severe' (clinical) category $(\mathrm{T} \geq 76)$ and $16.5 \%$ in the 'mild to moderate' category $(\mathrm{T} \geq 60$ ), leading to a total prevalence rate of $25.2 \%$. For the ADOS a total percentage of $18.8 \%$ was found, with $10.2 \%$ of the children having an autism classification and $8.6 \%$ of the children met criteria for an ASD classification. These percentages are lower than the percentages reported earlier with in-depth assessments (Garg et al. 2013; Plasschaert et al. 2015).

\section{Screening Accuracy}

Sensitivity, specificity, positive predictive (PPV) and negative predictive values (NPV) were computed to assess the screening accuracy of the ADOS' and SRS' classifications
Table 2 Sensitivity, specificity, positive predictive value (PPV) and negative predictive value (NPV) of the SRS parental report and ADOS classification compared to DSM-IV classification

\begin{tabular}{lllll}
\hline & Sensitivity & Specificity & PPV & NPV \\
\hline SRS class. $T \geq 60$ & .72 & .82 & .35 & .96 \\
SRS class. $T \geq 76$ & .46 & .97 & .63 & .95 \\
ADOS class & .64 & .89 & .45 & .95 \\
ADOS class. + SRS class. & .46 & .98 & .71 & .93 \\
$\quad T \geq 60$ & & & & \\
ADOS class. + SRS class. & .27 & .99 & .75 & .91 \\
T $\geq 76$ & & & & \\
\hline
\end{tabular}

in relation to a DSM-IV ASD diagnosis. The results are displayed in Table 2 .

With an SRS total T-score cutoff of $\mathrm{T} \geq 60$ (subclinical), the sensitivity in relation to the DSM-IV diagnosis was fair (.72) and the specificity was good (.82). With a cutoff of $\mathrm{T} \geq 76$ (clinical), sensitivity was poor (.46) and specificity was excellent (.97). In this population of children with NF1, the PPV was poor (.35) when using the SRS cutoff of $\mathrm{T} \geq 60$, demonstrating a low probability that a child with NF1 with a positive ( $\mathrm{T} \geq 60$ ) SRS score is being identified as having ASD according to the DSM-IV. Using this same cutoff of $\mathrm{T} \geq 60$, the NPV was excellent (.96), indicating a high probability that a child with NF1 with a negative score $(T<60)$ on the SRS is correctly identified as not having ASD according to the DSM-IV. Using the more stringent (clinical) cutoff of $\mathrm{T} \geq 76$, the PPV was poor (.63) and the NPV was excellent (.95).

For the ADOS classification, the sensitivity (.64) was poor and the specificity was good (.89). In this population of children with NF1, the PPV (.45) was poor, demonstrating a low probability that a child with NF1 with an ADOS classification is being identified as having ASD according to the DSM-IV. The NPV was excellent (.95), indicating a high probability that a child with NF1 without an ADOS classification is correctly identified as not having ASD according to the DSM-IV.

The sensitivity of the combined classification (classified with ASD on both the ADOS and SRS) was poor using both the $\mathrm{T} \geq 60$ and $\mathrm{T} \geq 76$ SRS T-score cutoffs (.46 and .27 , respectively). The specificity for both combined classifications was excellent (.98 and .99, respectively). In this population of children with NF1, for both combined classifications the PPV was fair (.71 and .75, respectively) and the NPV was excellent (.93 and .91, respectively).

In order to explain the finding of the substantially increased PPV when using the combined classification, an in-depth examination of the distribution of ADOS and SRS scores was performed. The number of participants with an SRS classification (for both $\mathrm{T} \geq 60$ and $\mathrm{T} \geq 76$ ) and ADOS classification were compared to the number 
Table 3 Percentage agreement in ASD classifications between ADOS and SRS

\begin{tabular}{llc}
\hline & \multicolumn{2}{l}{ ADOS } \\
\cline { 2 - 3 } & Yes & No \\
\hline SRS T $\geq 60$ & & \\
Yes & $8.7 \%(9 / 103)$ & $16.5 \%(17 / 103)$ \\
No & $8.7 \%(9 / 103)$ & $66.0 \%(68 / 103)$ \\
SRS T $\geq 76$ & & \\
Yes & $4.9 \%(5 / 103)$ & $3.9 \%(4 / 103)$ \\
No & $12.6 \%(13 / 103)$ & $78.6 \%(81 / 103)$ \\
\hline
\end{tabular}

of participants with a classification on both instruments. The results are displayed in Table 3 . As can be seen, the percentage of classification agreement is low for both the ADOS and SRS $\mathrm{T} \geq 60$ and the ADOS and SRS $\mathrm{T} \geq 76$ (i.e. $8.7 \%$ and $4.9 \%$ ). Figure 1 demonstrates that the SRS and ADOS both classify and fail to classify unique cases: a number of children with a SRS score of $\mathrm{T} \geq 60$ or $\mathrm{T} \geq 76$ are classified by the ADOS as 'non-spectrum' and a number of children with an ADOS ASD classification had a SRS T-score below 60.
Table 4 Correlates DSM-IV diagnosis

\begin{tabular}{llllll}
\hline & $t$ & df & $p$ & $\chi^{2}$ & $p$ \\
\hline Gender & - & - & - & 4.05 & .044 \\
Full-scale IQ & 1.09 & 111 & .28 & - & - \\
Verbal IQ & .62 & 112 & .53 & - & - \\
Performance IQ & 1.33 & 113 & .19 & - & - \\
Age & -2.36 & 116 & .02 & - & - \\
\hline
\end{tabular}

\section{Correlates}

There were no significant differences in full-scale, verbal, and performance intelligence scores between the group of children with and without DSM-IV ASD diagnosis (Table 2). Significantly more boys $(N=11)$ were clinically diagnosed with ASD according to the DSM-IV criteria compared to girls $(N=3)$. The group of children with a clinical ASD diagnosis was significantly older (mean age $=6.36$ ) compared to the group without diagnosis (mean age $=5.13$ ) (Table 4).

\section{Discussion}

The present study aimed to examine the prevalence of ASD in a sample of children with NF1 without a presumption of autistic symptoms. In our cohort of children with NF1,
Fig. 1 Scatterplot illustrating the distribution of SRS total T-scores (dotted lines indicate the SRS total cutoff scores $T \geq 60$ and $T \geq 76$ ) for the two ADOS categories (non-spectrum and ASD), demonstrating low agreement between the instruments

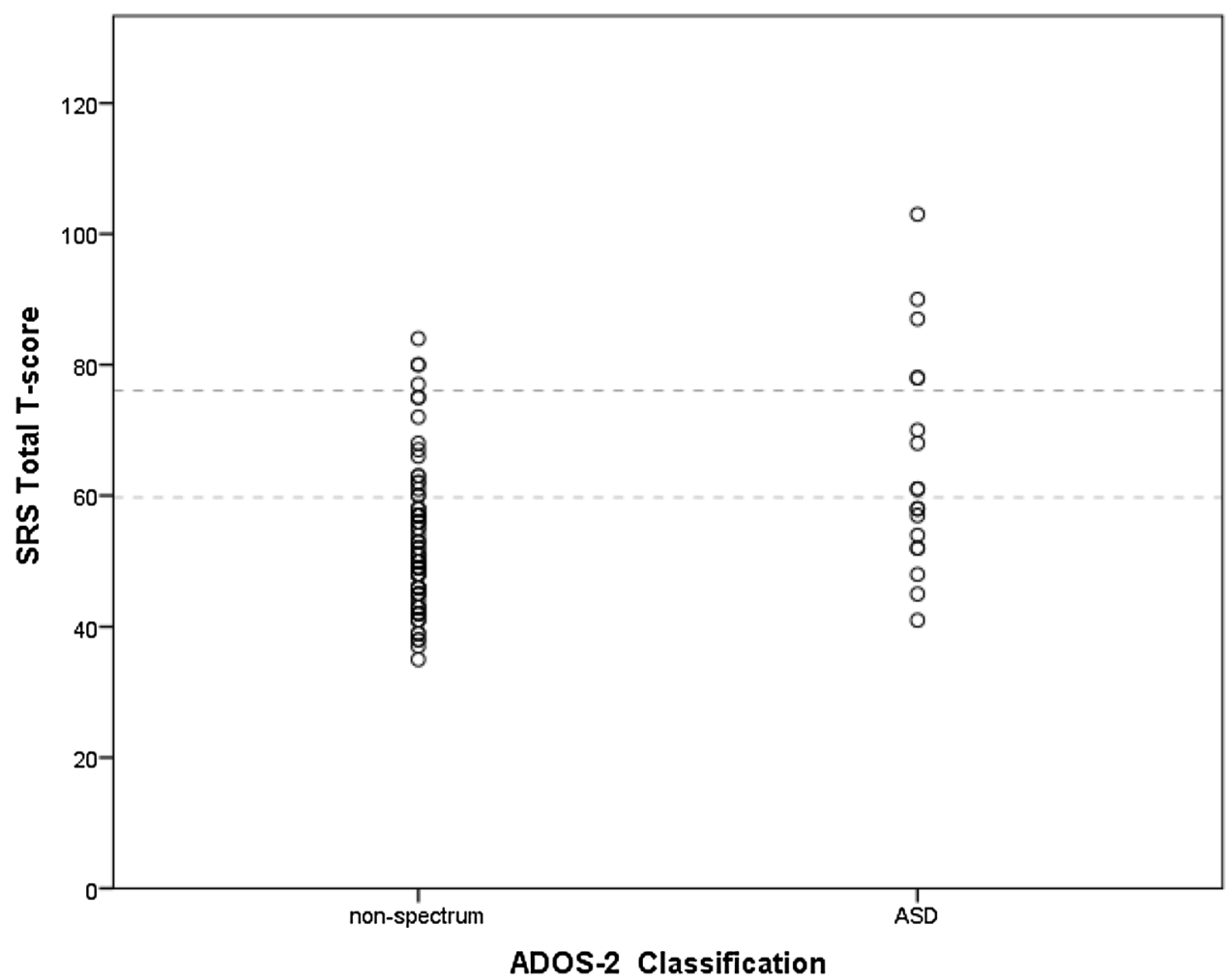


we found a DSM-IV based ASD prevalence rate of $10.9 \%$. Secondly, we aimed to assess the predictive value of a screening-based and observational instrument on the clinical DSM-IV ASD diagnosis. In this population of children with NF1, combining the classifications from both instruments yielded the highest predictive value on DSM-IV diagnosis. Thirdly, we examined several possible correlates and found a significant effect of gender and age on DSM-IV ASD diagnosis. Our reported DSM-IV based ASD prevalence rate of $10.9 \%$ endorses previous reports of an increased prevalence rate in children with NF1 as compared to the general population prevalence of about $0.8 \%$ (Baxter et al. 2015), and confirms the involvement of the various mutations leading to NF1 in the development of ASD (Morris et al. 2016). To our knowledge, this is the first study in which ASD prevalence is assessed in a sample of children with NF1 without an initial presumption of ASD. The prevalence reported in this study is likely more representative and accurate for the general pediatric NF1 population than reported by previous studies (Garg et al. 2013; Plasschaert et al. 2015).

Earlier reports of ASD prevalence rates have reported screening-based prevalence rates from 13 to 33\% (Adviento et al. 2014; Constantino et al. 2015; Garg et al. 2013; Van Eeghen et al. 2013; Walsh et al. 2013) and prevalence rates based on a observational instrument of 25-26\% (Garg et al. 2013; Plasschaert et al. 2015). The results from the observational- and screening instrument demonstrate that not all children with a classification on one of the instruments receive an eventual DSM-IV diagnosis. This implies that a group of children is present with subclinical ASD symptoms in the sample studied here. The percentage of children with screening-based subclinical ASD symptoms (i.e. 16.5\%) found in this study supports this. The question for future research remains whether this group of children with NF1 with subclinical ASD symptoms will receive a DSM-IV ASD diagnosis at a later age.

The substantial increase in PPV for the combination of the instruments' classification scores demonstrates the complementing effect of the two instruments in predicting DSM-IV ASD diagnosis. This might be explained by the difference in the assessment methods (e.g. informant and situation) of the instruments. In case of the SRS, one of the primary caregivers of the child acts as informant for ASD symptoms, whereas in case of the ADOS a trained and certified clinician observes and scores the behavior of the child. Different informants provide valuable and unique information with regard to ASD symptomatology, due to discrepancies in perspective and context, as demonstrated by Duvekot et al. (2015). Moreover, the ADOS assessment is performed in a semi-structured observational lab-setting, whereas the SRS assesses the child's behavior in daily life. The instruments provide unique information concerning autistic symptoms in the child; the classification agreement between the instruments is low (Table 3) and both instruments classify and fail to classify unique cases (Fig. 1). This underlines the importance of combining the findings from both instruments for ASD assessment in clinical practice.

The group of children with a DSM-IV ASD diagnosis was significantly older compared to the group without diagnosis. It has been demonstrated that children with ASD and other developmental, psychiatric, or neurologic comorbidities are usually diagnosed with ASD at a later age, possibly because the ASD symptoms are 'masked' by the comorbidities (Levy et al. 2010) or because assessment and treatment of somatic complaints are prioritized. As a consequence, our sample of children with NF1 might receive a DSM-IV ASD diagnosis at a later age or receive subclinical scores.

Intelligence scores were not related to the DSM-IV ASD diagnosis. This could be due to the lack of dispersion in intelligence scores in our sample, resulting in a homogenous sample regarding cognitive functioning. Examination of gender effects demonstrated an increased number of boys with a DSM-IV ASD diagnosis. This result is in line with the male predominance of ASD in the general population and the presumed female protective effect (Halladay et al. 2015) and with reports of higher prevalence rates of ASD in boys with NF1 (Garg et al. 2016).

A limitation in the current study is that SRS was not available in all children in this study. The parental response rate of $80 \%$ might have provided skewed results, since the exact reason for not completing the SRS questionnaire is unknown. However, there were no significant differences between the group of children from responders and nonresponders regarding gender, intelligence scores, age, DSMIV ASD diagnosis or ADOS total CSS, ADOS social affect CSS and ADOS restricted/repetitive behaviors CSS, thus it seems unlikely that differences in SRS scores between responders and non-responders would have been present. Secondly, the combination of the ADOS and ADI-R are generally advocated in ASD research, but the ADI-R was not included in the current study. The data in the current study was initially collected for patient care and the implementation of the ADI-R would have been too time-consuming. Instead, a thorough intake and heteroanamnestic interview with the parents was conducted. Thirdly, the sample used in this study included a number of two- and three year old children and ASD is often diagnosed at a later age. However, the instruments used in the current study are valid instruments for the assessment of autism and autism spectrum disorders in young children. Fourthly, since the data was collected from 2011 onwards, the ASD diagnoses were DSMIV based instead of DSM-V. Finally, although all children within ENCORE with NF1 were referred to the Department of Child and Adolescent Psychiatry/Psychology for neuropsychological evaluation and assessment of autistic symptomatology, a clinical referral bias might still be present in 
our sample. The problems experienced in children with NF1 are highly variable, and the severities of the difficulties fluctuate across the population (Lehtonen et al. 2013). Parents of children with for example more severe physical difficulties or developmental delays might shift their focus to these more pressing problems and postpone neuropsychological and behavioral assessment. At the same time, parents of children who experience limited to no difficulties might not see the need for neuropsychological or behavioral assessment. Nevertheless, the sample studied here is a key strength of the study; to our knowledge, this is the first study in which an unselected cohort of children with NF1 is referred for assessment of autistic symptomatology, regardless of an ASD presumption. The relatively large sample size of children with NF1 further strengthens our results. All children underwent uniform neuropsychological and ASD assessments, enabling unique comparisons between different instruments for ASD symptoms and comparison with the eventual DSM-IV ASD diagnosis, as well as the examination of potential correlates with the diagnosis.

\section{Conclusion}

A DSM-IV ASD prevalence rate of $10.9 \%$ demonstrates that the prevalence of ASD symptoms in children with NF1 is considerably higher compared to the general population, hereby emphasizing the importance of ASD assessment in this population. Our results underline the relevance of the use of multiple instruments (screening- and observational) for clinicians in order to correctly identify as many individuals with NF1 with ASD as needed. In addition to the group of children with a diagnosis, a substantial group of children with subclinical ASD symptoms is present as well, as was demonstrated by the screening- and observational instrument. This demonstrates the necessity to structurally follow the development of children with NF1.

Acknowledgments This research was financially supported by the Sophia Children's Hospital Fund (Rotterdam, the Netherlands) under Grant Number SSWO B14-02. Funders were not involved in the design of the study, nor in data collection, analysis, interpretation or writing the manuscript.

Author Contributions SE wrote the manuscript with support from all the authors. Supervision was carried out by JSL and SEM. Analyses were performed and supervised by SE, JSL, SEM, BD, and PFAN. GCD, ABR, LWH, RM, YE, CECB, RO contributed to the interpretation of the results. All authors discussed the results and contributed to the final manuscript.

\section{Compliance with Ethical Standards}

Conflict of interest The authors declare that they have no conflict of interest.

Ethical Approval All procedures performed in studies involving human participants were in accordance with the ethical standards of the institutional and/or national research committee and with the 1964 Helsinki declaration and its later amendments or comparable ethical standards.

Informed Consent This retrospective study was approved by the Medical Ethics Committee of the Erasmus Medical Center, the Netherlands (MEC-2015-203). Written informed consent was formally waived as there is no patient burden and no privacy concern.

Open Access This article is distributed under the terms of the Creative Commons Attribution 4.0 International License (http://creativeco mmons.org/licenses/by/4.0/), which permits unrestricted use, distribution, and reproduction in any medium, provided you give appropriate credit to the original author(s) and the source, provide a link to the Creative Commons license, and indicate if changes were made.

\section{References}

Adviento, B., Corbin, I. L., Widjaja, F., Desachy, G., Enrique, N., Rosser, T., ... Weiss, L. A. (2014). Autism traits in the RASopathies. Journal of Medical Genetics, 51(1), 10-20.

Barton, B., \& North, K. (2004). Social skills of children with neurofibromatosis type 1. Developmental Medicine \& Child Neurology, 46(8), 553-563.

Baxter, A. J., Brugha, T. S., Erskine, H. E., Scheurer, R. W., Vos, T., \& Scott, J. G. (2015). The epidemiology and global burden of autism spectrum disorders. Psychological Medicine, 45(3), 601-613.

Bayley, N. (2006). Bayley scales of infant and toddler development (3 edn.). San Antonio, TX: Harcourt Assessment.

Cicchetti, D. V. (2001). Methodological commentary the precision of reliability and validity estimates re-visited: Distinguishing between clinical and statistical significance of sample size requirements. Journal of Clinical and Experimental Neuropsychology, 23(5), 695-700. https://doi.org/10.1076/jcen.23.5.695.1249.

Constantino, J. N., Davis, S. A., Todd, R. D., Schindler, M. K., Gross, M. M., Brophy, S. L., ... Reich, W. (2003). Validation of a brief quantitative measure of autistic traits: comparison of the social responsiveness scale with the autism diagnostic interview-revised. Journal of Autism and Developmental Disorders, 33(4), 427-433.

Constantino, J. N., \& Gruber, C. P. (2015). Screeningslijst voor autismespectrumstoornissen (H. Roeyers, M. Thys, C. Druart, M. de Schryver \& M. Schittekatte, Trans.). Amsterdam: Hogrefe Uitgevers B.V.

Constantino, J. N., Zhang, Y., Holzhauer, K., Sant, S., Long, K., Vallorani, A., ... Gutmann, D. H. (2015). Distribution and withinfamily specificity of quantitative autistic traits in patients with neurofibromatosis type I. Journal of Pediatrics, 167(3), 621-+. https://doi.org/10.1016/j.jpeds.2015.04.075.

Duvekot, J., Van der Ende, J., Verhulst, F. C., \& Greaves-Lord, K. (2015). The screening accuracy of the parent and teacher-reported social responsiveness scale (SRS): Comparison with the 3Di and ADOS. Journal of Autism and Developmental Disorders, 45(6), 1658-1672. https://doi.org/10.1007/s10803-014-2323-3.

Garg, S., Green, J., Leadbitter, K., Emsley, R., Lehtonen, A., Evans, D. G., \& Huson, S. M. (2013). Neurofibromatosis type 1 and autism 
spectrum disorder. Pediatrics, 132(6), e1642-e1648. https://doi. org/10.1542/peds.2013-1868.

Garg, S., Heuvelman, H., Huson, S., Tobin, H., \& Green, J. (2016). Sex bias in autism spectrum disorder in neurofibromatosis type 1. Journal of Neurodevelopmental Disorders, 8(1), 1-8. https:// doi.org/10.1186/s11689-016-9159-4.

Garg, S., Lehtonen, A., Huson, S. M., Emsley, R., Trump, D., Evans, D. G., \& Green, J. (2013). Autism and other psychiatric comorbidity in neurofibromatosis type 1: Evidence from a populationbased study. Developmental Medicine \& Child Neurology, 55(2), 139-145.

Gotham, K., Pickles, A., \& Lord, C. (2009). Standardizing ADOS scores for a measure of severity in autism spectrum disorders. Journal of Autism and Developmental Disorders, 39(5), 693-705. https://doi.org/10.1007/s10803-008-0674-3.

Gotham, K., Risi, S., Pickles, A., \& Lord, C. (2007). The autism diagnostic observation schedule: revised algorithms for improved diagnostic validity. Journal of Autism and Developmental Disorders, 37(4), 613-627. https://doi.org/10.1007/s10803-006-0280-1.

Hachon, C., Iannuzzi, S., \& Chaix, Y. (2011). Behavioural and cognitive phenotypes in children with neurofibromatosis type 1 (NF1): The link with the neurobiological level. Brain \& Development, 33(1), 52-61.

Halladay, A. K., Bishop, S., Constantino, J. N., Daniels, A. M., Koenig, K., Palmer, K., ... Szatmari, P. (2015). Sex and gender differences in autism spectrum disorder: summarizing evidence gaps and identifying emerging areas of priority. Molecular Autism, 6(1), 1-5. https://doi.org/10.1186/s13229-015-0019-y.

Huijbregts, S. C., \& De Sonneville, L. M. (2011). Does cognitive impairment explain behavioral and social problems of children with neurofibromatosis type 1? Behavior Genetics, 41(3), 430436. https://doi.org/10.1007/s10519-010-9430-5.

Huijbregts, S. C., Jahja, R., De Sonneville, L., De Breij, S., \& SwaabBarneveld, H. (2010). Social information processing in children and adolescents with neurofibromatosis type 1. Developmental Medicine \& Child Neurology, 52(7), 620-625. https://doi.org/10 $.1111 / \mathrm{j} .1469-8749.2010 .03639 . x$.

Hus, V., Gotham, K., \& Lord, C. (2014). Standardizing ADOS domain scores: Separating severity of social affect and restricted and repetitive behaviors. Journal of Autism and Developmental Disorders, 44(10), 2400-2412. https://doi.org/10.1007/s1080 3-012-1719-1.

Hus, V., \& Lord, C. (2014). The autism diagnostic observation schedule, module 4: Revised algorithm and standardized severity scores. Journal of Autism and Developmental Disorders, 44(8), 1996-2012. https://doi.org/10.1007/s10803-014-2080-3.

Lehtonen, A., Howie, E., Trump, D., \& Huson, S. M. (2013). Behaviour in children with neurofibromatosis type 1: Cognition, executive function, attention, emotion, and social competence. Developmental Medicine \& Child Neurology, 55(2), 111-125. https:// doi.org/10.1111/j.1469-8749.2012.04399.x.

Levy, S. E., Giarelli, E., Lee, L. C., Schieve, L. A., Kirby, R. S., Cunniff, C., ... Rice, C. E. (2010). Autism spectrum disorder and co-occurring developmental, psychiatric, and medical conditions among children in multiple populations of the United States. Journal of Developmental \& Behavioral Pediatrics, 31(4), 267-275.
Lord, C., Rutter, M., DiLavore, P. C., \& Risi, S. (1999). Autism Diagnostic Observation Schedule (ADOS): Manual. Los Angeles: Western Psychological Services.

Lord, C., Rutter, M., DiLavore, P. C., Risi, S., Gotham, K., \& Bishop, L. S. (2012). Autism diagnostic observation schedule, second edition (ADOS-2): Manual (Part I). Los Angeles: Western Psychological Services.

Messiaen, L. M., Callens, T., Mortier, G., Beyens, D., Vandenbroucke, I., Van Roy, N., ... Paepe, A. D. (2000). Exhaustive mutation analysis of the NF1 gene allows identification of $95 \%$ of mutations and reveals a high frequency of unusual splicing defects. Human Mutation, 15(6), 541-555.

Morris, S. M., Acosta, M. T., Garg, S., Green, J., Huson, S., Legius, E., ... Constantino, J. N. (2016). Disease burden and symptom structure of autism in neurofibromatosis type 1: A study of the international nf1-asd consortium team (INFACT). JAMA Psychiatry. https://doi.org/10.1001/jamapsychiatry.2016.2600.

Noll, R. B., Reiter-Purtill, J., Moore, B. D., Schorry, E. K., Lovell, A. M., Vannatta, K., \& Gerhardt, C. A. (2007). Social, emotional, and behavioral functioning of children with NF1. American Journal of Medical Genetics Part A, 143A(19), 2261-2273. https://doi. org/10.1002/ajmg.a.31923.

Plasschaert, E., Descheemaeker, M. J., Van Eylen, L., Noens, I., Steyaert, J., \& Legius, E. (2015). Prevalence of autism spectrum disorder symptoms in children with neurofibromatosis type 1 . American Journal of Medical Genetics Part B Neuropsychiatric Genetics, 168B(1), 72-80.

Rauen, K. A. (2013). The RASopathies. Annual Review of Genomics and Human Genetics, 14, 355-369. https://doi.org/10.1146/annur ev-genom-091212-153523.

Van Eeghen, A. M., Pulsifer, M. B., Merker, V. L., Neumeyer, A. M., van Eeghen, E. E., Thibert, R. L., \& ... Thiele, E. A. (2013). Understanding relationships between autism, intelligence, and epilepsy: A cross-disorder approach. Developmental Medicine \& Child Neurology, 55(2), 146-153. https://doi.org/10.1111/ dmen.12044.

Van Minkelen, R., Van Bever, Y., Kromosoeto, J. N., Withagen-Hermans, C. J., Nieuwlaat, A., Halley, D. J., \& Van Den Ouweland, A. M. (2014). A clinical and genetic overview of 18 years neurofibromatosis type 1 molecular diagnostics in the Netherlands. Clinical Genetics, 85(4), 318-327.

Walsh, K. S., Velez, J. I., Kardel, P. G., Imas, D. M., Muenke, M., Packer, R. J., ... Acosta, M. T. (2013). Symptomatology of autism spectrum disorder in a population with neurofibromatosis type 1. Developmental Medicine \& Child Neurology, 55(2), 131-138.

Wechsler, D. (1991). Wechsler intelligence scale for children (3 edn.). San Antonio, TX: Psychological Corporation.

Wechsler, D. (2002). Wechsler preschool and primary scale of intelligence (3 edn.). San Antonio, TX: Psychological Corporation.

Wechsler, D., \& Naglieri, J. A. (2006). Wechsler nonverbal scale of ability (WNV). San Antonio, TX: Harcourt Assessment.

Williams, V. C., Lucas, J., Babcock, M. A., Gutmann, D. H., Korf, B., \& Maria, B. L. (2009). Neurofibromatosis type 1 revisited. Pediatrics, 123(1), 124-133. https://doi.org/10.1542/peds.2007-3204. 